East African Medical Journal Vol. 80 No. 12 December 2003

PREVALENCE AND CLINICAL PRESENTATION OF HIV INFECTION AMONG NEWLY HOSPITALISED SURGICAL PATIENTS AT MUHIMBILI NATIONAL HOSPITAL, DAR ES SALAAM, TANZANIA

C. Mkony, MD, MMed, Department of Surgery, G. Kwesigabo, MD, MMed, PhD, Department of Epidemiology/Biostatistics, E. Lyamuya, MD, MMed, PhD, Department of Microbiology/lmmunology and F. Mhalu, MBchB, MRCPath, Department Microbiology/lmmunology, Muhimbili University College of Health Sciences, P.O Box 65001, Dar es Salaam, Tanzania

Request for reprints to: Prof. C. A. Mkony, Department of Surgery, Muhimbili University College of Health Sciences, P. O. Box 65001, Dar es Salaam, Tanzania

\title{
PREVALENCE AND CLINICAL PRESENTATION OF HIV INFECTION AMONG NEWLY HOSPITALISED SURGICAL PATIENTS AT MUHIMBILI NATIONAL HOSPITAL, DAR ES SALAAM, TANZANIA
}

\author{
C. MKONY, G. KWESIGABO, E. LYAMUYA and F. MHALU
}

\begin{abstract}
Background: In Tanzania information is lacking on the prevalence of HIV infection in surgical patients in tertiary care facilities, in whom there are many points of special interest.

Objective: To determine the prevalence of HIV infection and associated clinical and demographic features among hospitalised surgical patients at Muhimbili National Hospital (MNH).

Setting: Muhimbili National Hospital.

Materials and Methods: Consecutive newly admitted patients were tested for HIV antibodies after pre-test counselling. Sera were tested using a dual ELISA algorithm. The data were analysed to determine the prevalence of HIV infection and relationships of serostatus with clinical and socio-demographic characteristics.

Results: Of 1,534 patients admitted during the study, 1,031(67.2\%) consented to HIV testing following pre-test counselling. The prevalence of AIDS-related clinical features in patients who declined to be HIV tested was similar to that of seronegative patients, but significantly lower than that of seropositive patients. The overall age-adjusted HIV prevalence was $10.5 \%(95 \% \mathrm{Cl}=9.9-14.0)$. The highest age-specific $\mathrm{HIV}$ prevalence was in the age group 35-44 years at $27.9 \%$. No one was infected in the age group 0-4 years $(n=111)$. Differences in prevalence between age groups were statistically significant $(\mathbf{p}<0.0001)$. Patients with granulomatous and suppurative infections had HIV prevalence of $28.3 \%$. Twenty of 124 seropositive patients $(\mathbf{1 6 . 1 \% )}$ died in hospital compared to 58 of $907(6.4 \%)$ of seronegative patients $(\mathrm{p}=0.0001)$.

Conclusion: At Muhumbili National Hospital overall HIV prevalence in hospitalised surgical patients were $10.5 \%$, compared to an overall national prevalence of $6.7 \%$. Patients in the age groups 25 to 34 and 35 to 44 years had HIV seroprevalence of $26.8 \%$ and $27.9 \%$ respectively. Patients with infective conditions had the highest HIV prevalence. HIV seropositive patients were associated with higher hospital mortality than seronegative patients.
\end{abstract}

\section{INTRODUCTION}

Since the first three cases of AIDS were identified in Kagera Region, Northwestern Tanzania in 1983, a rapid and relentless spread has taken the disease into every corner of the country. In 2001 the number of reported AIDS cases was 14,112, leading to projected countrywide cases at 71,000(1). Estimates of HIV infection rates in the population are based on surveillance of antenatal clinic attendees, blood donors and patients of sexually transmitted disease clinics. In 2001 the HIV seroprevalence among blood donors countrywide was estimated at $11.01 \%(1)$. Of patients attending sexually transmitted diseases (STDs) clinic in Dar es Salaam, $44.2 \%$ of those who consented to HIV testing were positive(2). A study involving Police officers in Dar es Salaam revealed HIV prevalence of $18 \%$ in females and $13.3 \%$ in males(3). The seroprevalence among 800,000 women going to deliver at various health care facilities in the country was $13.3 \%(2)$. Of consecutive febrile patients admitted to the medical wards at $\mathrm{MNH}$ in $1995,55 \%$ were infected with $\operatorname{HIV}(4)$

The seroprevalence in blood donors is probably an under-representation of the seroprevalence in the general population because those who have the courage to "volunteer" to donate blood, where they know HIV screening will be done, may be a group with low risk factors for HIV infection. However, it is reasonable to assume that the nation-wide prevalence of HIV infection in the adult population during 2001-2002 will not exceed $13.3 \%$, the seroprevalence in young sexually active women going to deliver at different health care facilities. Tanzania has an estimated 2.2 million persons of all ages living with HIV/AIDS(1). With a national 
population of 33 million people, the overall prevalence of HIV infection nationally is therefore $6.7 \%$. There are marked differences in HIV seroprevalence in different parts of Tanzania, and among different population groups. It has been stated elsewhere in the past that urban prevalence rates are up to ten times higher than rural rates(5). Collectively, the findings from these studies would seem to suggest a current prevalence of HIV infection nation-wide in adults of just over $10 \%$. The Adult Morbidity and Mortality Study done in three settings in Tanzania has shown that HIV/AIDS is the leading cause of death among men aged 15 -59 years in two of the three study areas(6).

HIV infection has been studied in different clinical settings at MNH. In hospitalised paediatric patients in a 1995-1996 study, the HIV seroprevalence was $19.2 \%(7)$. In medical in-patients a seroprevalence of $35 \%$ in males and $38 \%$ in females was reported in 1992(8). In Obstetric patients at the antenatal clinic the seroprevalence in a 1996 -1998 study was $13.7 \%(9)$ and in psychiatric in-patients the seroprevalence was estimated at 22\%(10). To date there has been no similar study in general surgery. HIV infection in surgical patients is a matter of special interest because of its influence on the clinical presentation of various surgical diseases, the increased prevalence of conditions like atypical Kaposi's sarcoma, lymphomas, pyomyositis, empyema thoracis and various malignancies in HIV infected persons. It has been suggested that prostate cancer may be more common in patients with HIV/ AIDS than in the general population(11). Aggressive cutaneous squamous cell carcinomas have been reported in young HIV-infected people(12).

The potential risk of transmission from patients to staff is another area of considerable interest and controversy. Many diagnostic and therapeutic manoeuvres in clinical surgery carry a potential for contamination and infection of staff by blood and other body fluids that has no parallel in the non-surgical medical disciplines(13). Blood and potentially contaminated body fluids in urological surgery and the risk they pose to the surgeon deserve special attention(14). The range of attitudes of surgeons towards HIV infection in patients and health care workers (HCW's) is wide, and opinions vary, including surgeons' refusal to operate on seropositive patients and recommendation to bar infected surgeons from operating. Considerable controversy exists on the risk of seroconversion in the absence of antiretroviral prophylaxis in the HCW following a solid needle-stick injury during surgery on an HIV infected patient, but it is believed to be of the order of $0.3 \%(13,15)$.

A Democratic Republic of Congo study showing similar trends of infection in surgical, nursing and administrative staff at the Mama Yemo Teaching Hospital supports the conclusion that the risk of transmission from patient to surgeon is low(15). The patient's viral load, which depends on the disease stage, is one of the factors influencing this risk $(13,16)$.

It is therefore of interest to study the prevalence of HIV infection and the pattern of presentation in surgical patients at this point in time in the course of the epidemic in this area of high HIV prevalence.

Objectives: To determine the prevalence of HIV infection in newly hospitalised surgical patients at Muhimbili National Hospital (MNH), its relationship to various socio-demographic features of the patients and to various surgical conditions.

\section{MATERIALS AND METHODS}

Study subjects: All new patients being admitted to the paediatric (1-10 years of age) and adult surgical wards between December 2001 and February 2002 were recruited into the study. The majority of MNH admissions originated from Dar es Salaam (a city of about 3 million inhabitants) and its surroundings, but some came as referrals from upcountry. MNH has 1400 beds, of which 230 are for Paediatric and adult General Surgery.

Demographic and clinical Bio-data collection: On admission patients were informed of the ongoing study, enrolled, interviewed and examined by an attending doctor. A nurse with training and experience in HIV counselling privately counselled each patient for HIV testing. In the case of children or unconscious patients a parent or accompanying person was interviewed. The information recorded on a questionnaire for each patient included the patient's personal particulars, relevant symptoms and signs, medical history, education level, marital status, discharge diagnosis and final disposal of the patient. The record also indicated whether the patient consented to HIV testing, the test result for those who consented, and results of sputum examination for acid fast bacteria.

HIV testing: Consenting patients had blood drawn for HIV serology. HIV testing was by dual ELISA strategy done sequentially. The first ELISA was Enzygnost anti HIV1/2 Plus (Dade Behring, Marburg, Germany) and the second ELISA was Welcozyme HIV Recombinant (Abbott Murex, Dartford, UK). Samples reactive on both assays were considered seropositive for HIV antibodies. Samples that were non reactive on the first assay were reported as HIV seronegative. Repeatedly discordant samples were confirmed by Western blot. Interpretation of Western blot reactivity was done according to the World Health Organization criteria(17).

The data were coded, entered, cleaned and analysed using EPIINFO software (Centres for Disease Control, Atlanta, Georgia) according to the study objectives. As individual diagnoses were found to be too numerous, diagnoses were combined into diagnostic groups to facilitate analysis. Differences between proportions were assessed using the $\mathrm{x}^{2}$ test, confidence intervals were calculated using the Fleiss quadratic approximation formula and are reported at $95 \%$ level.

Ethical issues: Written consent was obtained from all study subjects before recruitment. Pre-test counselling was done for those agreeing to HIV testing, followed by posttest counselling for those opting to receive their results. Ethical clearance for the study was obtained from the Muhimbili College Ethical Committee. HIV test results were handled confidentially. 


\section{RESULTS}

The study was conducted over three months from December 2001 to February 2002 during which time 1,534 patients were admitted to the surgical wards. Of these, 1030 were male $(67.1 \%)$ and 504 were female (32.9\%). Following counselling, 672 men (65.2\%) consented to HIV testing while 359 women $(71.2 \%)$ consented, giving an overall consent rate of $67.2 \%$.

Of 1031 patients who consented to HIV testing, $11 \%$ of the males were seropositive while $13.9 \%$ of female patients were seropositive $(p=0.1)$. The age adjusted prevalence of HIV infection was found to be $10.5 \%$.

The age and sex specific HIV seroprevalence is shown in (Figure 1). Below 24 years of age those infected were predominantly female, as were those above 55 years, while between 25 and 54 years of age, more males than females were infected. Even when the zero to four year age group (prevalence $=0$ ) is excluded, the difference in HIV prevalence between various age groups was statistically significant $(\mathrm{p}<$ $0.0001)$.

Of all patients above the age of 15 years who were tested for HIV antibodies $(\mathrm{n}=711)$, divorced and widowed patients had HIV seroprevalence rates of $20.6 \%$ and $18.4 \%$ respectively, compared to married and single patients whose HIV seroprevalence rates were $15.5 \%$ and $13.4 \%$ respectively. The difference in HIV seroprevalence between the different marital status groups were not statistically significant $(\mathrm{p}=0.5)$. No association was found between patient's education level and HIV status.

Of the 1031 patients who were tested for HIV antibodies, the HIV seroprevalence in Christians $(\mathrm{n}=589)$ and Moslems $(n=424)$, the main groups, were $12.3 \%$ and $11.1 \%$ respectively. The difference was not statistically significant $(\mathrm{p}=0.52)$.

Table 1 shows the prevalence of HIV infection in conditions traditionally associated with HIV infection/ AIDS. Patients presenting with stab wounds and those with breast cancer also had high prevalence of HIV infection.

Table 1

HIV Serostatus in selected diagnoses

HIV Serostatus

\begin{tabular}{lllll}
\hline Diagnosis & Total $(\mathrm{n})$ & No. positive & $\%$ positive & $95 \% \mathrm{Cl}$ \\
\hline Kaposi's sarcoma & 17 & 9 & 52.9 & $28.5-76.1$ \\
Pyomyositis & 21 & 7 & 33.3 & $15.4-56.8$ \\
Abscess & 11 & 6 & 54.5 & $24.5-81.6$ \\
Anal fissure & 8 & 5 & 62.5 & $25.8-89.7$ \\
Tb adenitis & 6 & 5 & 83.3 & $36.4-99.1$ \\
Empyema & 9 & 4 & 44.4 & $15.3-77.3$ \\
Peritonitis & 11 & 4 & 36.3 & $12.4-68.3$ \\
Breast cancer & 24 & 4 & 16.6 & $5.5-38.2$ \\
Stab wounds & 5 & 3 & 60 & $17.0-92.7$ \\
\hline
\end{tabular}

Table 2

HIV Serostatus by diagnostic group among hospitalised surgical patients

\begin{tabular}{lllll}
\hline \multirow{2}{*}{ Diag. Group } & Total number & No. positive & $\%$ positive & $95 \% \mathrm{Cl}$ \\
\hline Suppurative infections & 99 & 28 & 28.3 & $19.9-38.4$ \\
Other infections & 46 & 7 & 15.2 & $6.8-29.4$ \\
Malignancies & 222 & 27 & 12.2 & $8.3-17.4$ \\
Benign tumours & 29 & 2 & 6.9 & $1.2-24.2$ \\
Trauma & 214 & 13 & 6.1 & $3.4-10.4$ \\
Metabolic disorders & 48 & 8 & 16.7 & $7.9-30.7$ \\
Congenital anomalies & 38 & 0 & 0 & $0.0-11.4$ \\
G.I. conditions. & 105 & 16 & 15.2 & $9.2-23.9$ \\
Misc. abd. conditions & 82 & 7 & 8.5 & $3.7-17.3$ \\
Obstructive uropathies & 78 & 3 & 3.8 & $2.5-26.2$ \\
Other Urol. conditions & 32 & 5 & 15.6 & $5.8-33.5$ \\
Others & 43 & 8 & 6.5 & $8.9-33.9$ \\
\hline Total & 1031 & 124 & &
\end{tabular}

The prevalence of HIV infection among patients with suppurative and granulomatous infections was higher than that of other diagnostic groups $(\mathrm{p}<0.001)$. 
Table 3

Symptoms, signs and mortality according to HIV testing status and serostatus among hospitalised surgical patients

\begin{tabular}{lllll}
\hline Symptom/ Sign & $\begin{array}{l}\text { Seropositive }(\mathrm{n}=24) \\
\text { No. }(\%)\end{array}$ & $\begin{array}{l}\text { Seronegative }(\mathrm{n}=907) \\
\text { No. }(\%)\end{array}$ & $\begin{array}{l}\text { Untested }(\mathrm{n}=503) \\
\text { No. }(\%)\end{array}$ & $\begin{array}{l}\text { All }(\mathrm{n}=1534) \\
\text { No. }(\%)\end{array}$ \\
\hline $\begin{array}{l}\text { Symptoms } \\
\quad \text { Diarrhoea }\end{array}$ & $12(9.7)^{1}$ & & & \\
$\quad$ Productive cough & $13(10.5)^{2}$ & $19(2.1) 1$ & $10(2.0)$ & $41(2.7)$ \\
$\quad$ Fever & $26(21)^{3}$ & $36(4.0)^{2}$ & $22(4.4)$ & $71(4.6)$ \\
$\quad$ Weight Loss & $23(18.6)^{4}$ & $103(11.4)^{3}$ & $51(10.2)$ & $180(11.7)$ \\
Signs & $10(8.1)^{\mathrm{a}}$ & $10(9.8)^{4}$ & $25(5)$ & $137(8.9)$ \\
$\quad$ Oral thrush & $22(18)^{\mathrm{b}}$ & $10(1.1)^{\mathrm{a}}$ & $8(1.6)$ & $28(1.8)$ \\
$\quad$ Large nodes & $20(16.1)^{\mathrm{c}}$ & $55(6.1)^{\mathrm{b}}$ & $22(4.4)$ & $99(6.5)$ \\
$\quad$ M-P rash* & $20(16.1)$ & $58(6.4)$ & $20(4.0)$ & $67(4.4)$ \\
Mortality & & $20(4.0)$ & $98(6.4)$ \\
\hline
\end{tabular}

*M-P = Maculo-papular

p-value $1<0.001,2<0.013<0.001,4<0.000, \mathrm{a}<0.001, \mathrm{~b}<0.0001, \mathrm{c}<0.0001$

Figure 1

Age and Sex specific HIV prevalence among hospitalised surgical patients

\section{Figure 2}

Response to counselling for HIV testing by age groups among newly hospitalised surgical patients
Table 2 shows the serostatus of patients by diagnostic groups. When diagnoses were grouped into broad categories, the highest HIV prevalence was among patients with suppurative and granulomatous infections $(28.3 \%)$. The lowest prevalence was among patients with congenital anomalies $(0 \%)$, obstructive uropathies $(3.8 \%)$, and trauma $(6.1 \%)$.

Figure 2 demonstrates that between 15 and 34 years of age, which includes the majority of HIV infections, just over $50 \%$ of patients gave consent for HIV testing. In those below 15 years, in the majority of whom a parent or guardian was involved in counselling, and in whom HIV seroprevalence was low, over $80 \%$ gave consent. In patients aged 35 years or older the proportion consenting to testing increased with age from $62.4 \%$ to $72.9 \%$.

Table 3 shows a comparison of the prevalence of symptoms and signs associated with AIDS, and mortality in patients who were HIV tested, patients who declined to be tested, and in the study population as a whole. Patients who declined to be tested had features similar to those of HIV negative patients.

Sixteen patients with productive cough submitted three consecutive sputum specimens each for Z-N stain, and three of the patients were found to have smear positive sputum for acid-fast bacteria.

\section{DISCUSSION}

HIV disease has become a clinical, social, economic and public health problem of enormous proportions in many countries of the world, especially in sub-Saharan Africa. For this study the college ethical clearance committee required that patients be counselled before testing for HIV antibodies, unlike some years ago when testing could be done without having to get patients' consent, provided the unlinked anonymous strategy was adopted $(7,18)$. The $67.2 \%$ of patients who agreed to 
take the HIV test were a higher proportion than that in counselled clients nation-wide whereby of 6,539 counselled in 2000, only $51 \%$ consented to HIV testing(2).

In an earlier study in a rural setting in Northwestern Tanzania 55.9\% of those counselled agreed to be tested (18), while in a study of pregnant women attending antenatal clinics in Dar es Salaam $76.4 \%$ agreed to be tested. The higher testing rate in the latter study might be explained by the fact that the study was connected with an ongoing antiretroviral prophylaxis study to prevent mother to child HIV transmission(9).

HIV/AIDS in our socio-cultural setting continues to be highly stigmatised, and the need to improve counselling has been noted before $(9,19)$. The stigma around HIV/AIDS is further demonstrated by the fact that in the study by Kilewo et al, only $16.7 \%$ of the enrolled seropositive women were prepared to disclose their serostatus to their sexual partners(9). By comparison, at a San-Francisco hospital, $99.5 \%$ of hospitalised Orthopaedic patients consented to HIV antibody testing (20). However, the stigma of HIV infection is by no means confined to developing countries(21).

Most studies in Tanzania show peak prevalence of HIV infection in the age group 25-34 years or younger(1,2,18). In a study relating viral load to transmission in Uganda it was shown that the highest incidence of seroconversion was in the age group 15-19 years(22), which suggests peak prevalence in a younger age group than usual. These findings contrast with those in the present study in which peak prevalence was in the age group 35-44 years at 27.9\%, but the 25-34 year age group was a close second at $26.8 \%$ (Figure 1).

HIV transmission in this part of the world is known to be mainly by heterosexual intercourse $(1,2,22,23)$. A high seroprevalence in divorced and widowed patients may be due to increased high-risk sexual behaviour in adults forced by circumstances to be without their marriage partners. Widowed patients could, indeed, have lost their spouses to AIDS. However, the differences in seroprevalence between the different marital status groups did not reach statistical significance. A study in elderly medical patients at MNH also showed no difference in prevalence according to marital status. The same study showed no association in seroprevalence with urban versus rural residence, education level or socio-economic status(24).

Religious affiliation did not show any association with HIV serostatus. Presumably sexual behaviour is guided more by socio-cultural norms than religious and moral ethics.

The sample size for the study was determined under the assumption of a seroprevalence rate of 30$40 \%$ as seen in medical wards at MNH. The unexpectedly low seroprevalence led to the relatively small numbers of seropositive patients in any given clinical condition. With these limitations in mind the majority of patients with tuberculous cervical lymphadenitis, anal fissure or fistula, abscesses and Kaposi's sarcoma were seropositive, while there was also a high prevalence in patients with empyema thoracis, primary peritonitis, pyomyositis, and breast cancer. At first sight it may seem curious that patients presenting with stab wounds had high HIV prevalence. It can be postulated that these young individuals involve themselves in rows and other socially dangerous situations, and that the same character traits expose them to high risk sexual behaviour leading to HIV infection. A study by Amir et al(25) from Muhimbili National Hospital suggested that HIV seroprevalence was low in patients with breast cancer, a finding that is not supported by the results of the present study (Table 1).

Of $1031 \mathrm{HIV}$ tested patients, 20 of $124(16.1 \%)$ seropositive patients died in hospital while of 907 seronegative patients, 58(6.4\%) died in hospital $(\mathrm{p}=0.0001$, relative risk 2.5$)$. The mortality of seropositive surgical inpatients in this study is lower than that found in seropositive paediatric patients at MNH of $21.4 \%$ (7). Patients with suspected pulmonary tuberculosis without a surgical disease are normally taken into the medical wards. Thus a relatively small number of patients in this study were tested for acidfast bacteria and only three of them had positive smears.

It is tempting to speculate that the patients who declined to be tested for HIV infection were those at high risk of being HIV positive. However, the prevalence of diarrhoea, productive cough, fever and weight loss, symptoms that are common in AIDS patients, was comparable in patients who declined HIV testing and patients who were HIV seronegative, but significantly lower than in HIV seropositive patients.

As shown in Table 3, the same trend is observed when the prevalence of physical signs commonly found among patients with AIDS are compared. Comparison of the hospital mortality in the four groups again shows that the untested patients had similar mortality to the seronegative patients but that these were significantly lower than in the seropositive group $(\mathrm{p}<0.0001)$. These findings suggest that the patients who declined to undergo HIV-testing were simply patients who did not want to be bothered with another blood test and were not a high-risk group for HIV infection/AlDS.

\section{CONCLUSION}

In this study involving hospitalised surgical patients, the overall age-adjusted HIV seroprevalence of $10.5 \%$ was lower than expected in comparison to findings from similar studies in other hospital departments, but patients in the 14-25 years age groups had seroprevalence rates of $26.8-27.9 \%$. The majority of patients with tuberculous lymphadenitis, anal fissure or fistula, stab wounds, abscesses and Kaposi's sarcoma were HIV 
positive. Interestingly, patients who declined to be tested for HIV antibodies had clinical features comparable to the HIV negative patients. HIV positive patients had significantly higher hospital mortality than those who were HIV negative.

\section{ACKNOWLEDGEMENTS}

This study was supported by a research capacity grant from the Swedish Agency for Development Cooperation, SIDA/SAREC, through the Muhimbili University College of Health Sciences. We thank the hospital and college authorities for permission to carry out the study, and the medical, nursing and laboratory staff who participated in the study.

\section{REFERENCES}

1. HIV/AIDS/STI Surveillance Report, January-December 2001. Ministry of Health, Tanzania Mainland. Report Number 16.

2. HIV/AIDS/STI Surveillance Report, January - December 2000 Ministry of Health, Tanzania Mainland. Report Number 15.

3. Bakari, M., Lyamuya, E., Mugusi, F., et al. The prevalence and incidence of HIV-1 infection and syphilis in a cohort of police officers in Dar es Salaam, Tanzania: a potential population for HIV vaccine trials. AIDS 2000 14:313-320.

4. Archibald, L. K., den Dulk, M. O,. Pallangyo, K. J and Reller, L. B. Fatal Mycobactenum tuberculosis bloodstream infections in febrile hospitalised adults in Dar es Salaam, Tanzania. CID 1998; 26:290-296.

5. Friedland, G. H. and Kloin, R. S. Transmission of the Human Immunodeficiency Virus. N. Eng. J. Med. 1987; 317:1125-1135.

6. Policy implications of adult morbidity and mortality, end of phase 1 report. A bilateral health project funded by United Kingdom Department for International Development and Government of the United Republic of Tanzania. Ministry of Health, Dar es Salaam, Tanzania. August 1997.

7. Kawo, G., Karlsson, K., Lyamuya, E., T., et al. Prevalence of HIV Type 1 infection, Associated clinical features and mortality among hospitalised children in Dar es Salaam, Tanzania. Scan. J. Infect. Dis., 2000; 32:357-363.

8. Pallangyo, K., Thomas, A., Rusizoka, O., et al. Prevalence of HIV infection among medical admissions. Vll th International AIDS Conference, Younde, Cameroon, 1992; No. 29.

9. Kilewo, C., Massawe, A., Lyamuya, E., et al. HIV counseling and testing of pregnant women in sub-Saharan Africa: experiences from a study on prevention of mother to child transmission in Dar es Salaam, Tanzania. J. Acquir.
Immune. Def. Syndr. 2001; 28:458-462.

10. Hogan, N. M. and Kilonzo, G. P. Psychiatric Co-morbidity in medical patients with AIDS: Some considerations for clinicians and counselors in Tanzania. Tanzania. Med. J. 1997; 12:6-11.

11. Crum, N. F., Haule, B., Utz, G. and Wallace, M. Increased risk of prostate cancer in HIV infection? AIDS 2002; 16:1703-1704.

12. Nguyen, P., vin-Christian, K., Ming, M. E. and Berger, T. Aggressive squamous cell carcinoma in persons infected with Human Immunodeficiency Virus. Arch. Dermatol. 2002; 138:758-763.

13. Flum, D. R., and Wallack, M. K. The Surgeon's database for AlDS: A Collective review. J. Amer. Coll. Surg. 1997; 184:403-411.

14. Kapoor, D. A., Smiley, D. G., and Reddy, P. K. The risk of exposure to potentially contaminated body fluids in urological surgery. J. Urol. 1993; 149:1058-1060.

15. Cairns, J. The health worker and HIV in high-incidence poor countries: Part I HIV and the health worker. Trop. Doct. 2000; 30:175-177.

16. Cardo, D. M. A case - control study of HIV seroconversion. N. Engl. J. Med. 1997; 337:1485-1490.

17. Proposed WHO criteria for interpreting results of Western blot assays for HIV-1 and HIV-2, and HTLV-I and HTLVII. Wld. Hlth. Org. Weekly Epidem. Rec. 1990; 37:281-283.

18. Kwesigabo, G., Killewo, J. Z., Sandstrom, A., et al Prevalence of HIV infection among hospital patients in North West Tanzania. AIDS Care. 1999;11:87-93.

19. Killewo, J. Z., Kwesigabo, G., Comoro, C., et al. Acceptability of HIV testing with counseling in a rural village in Kagera, Tanzania. AIDS Care. 1998; 10:431439.

20. Paiement, G. D., Hymes, R. A., La Douceur, M. S., Gosselin, R. A. and Green, H. D. Postoperaive infections in asymptomatic HIV Seropositive orthopedic trauma patients. J. of Trauma. 1994; 37:545-551.

21. Herek, G. M., Capitanio, J. P. and Widaman, K. F. HIVrelated stigma and knowledge in the United States: Prevalence and trends. 1991-1999. Amer. J. Public Health 2002; 92:371-377.

22. Quinn, T. C., Wawer, M. J., Sewankambo, A. et al. Viral load and heterosexual transmission of Human Immunodeficiency Virus Type 1. New Eng. J. Med. 2000; 342:921-929

23. Ndosi, N. K. and Shao, J. F. Psychiatric patients presenting with HIV infection in Dar es Salaam, Tanzania. Tanzania Med. J. 1992; 7:119-21.

24. Mtei, L. N. and Pallangyo, K. P. HIV infection in elderly medical patients. East Afr. Med. J. 2001; 78:144-147.

25. Amir, H., Makwaya, C., Mhalu, F., Mbonde, M. P. and Schwartz-Albiez, R. Breast cancer during the HIV epidemic in an African population. Oncol Rep 2001; 8: 659-651. 